\title{
Research on the dynamic buckling of functionally graded material plates under conditions of one edge fixed and three edges simply supported
}

\author{
Wang XIN, Han Zhi JUN, Wu Ya LI \\ College of Mechanics, Taiyuan University Of Technology Taiyuan 030024, China
}

\begin{abstract}
Based on the strain assumption and linear mixing rate of Vogit, the physical property parameter expression of functionally graded material plates is obtained. According to the theory of small deformation and Hamilton principle, the dynamic buckling governing equation of functionally graded material plates under longitudinal load is obtained. Using the method of trial function, the analytical expression of critical load and the buckling solution of the functionally graded material plate under conditions of one edge fixed and three edges simply supported is obtained. The analytical expression of critical load is numerically calculated by METLAB. The influence of geometric size, gradient index, modal order and material composition on critical load is discussed. The results show that the critical buckling load decreases exponentially with the increase of critical length, decreases with the increase of gradient index k, increases with the increase of modal order, and the elastic modulus of constituent materials has significant effect on the critical load. The higher-order buckling modes of functionally graded material plates are prone to occur under the condition of high longitudinal load.
\end{abstract}

\section{Introduction}

Functionally graded materials are non-homogenous new composite materials that show smooth and continuous characteristics among different materials. Functionally graded materials can effectively avoid or reduce stress concentration without sudden changes in physical properties. Due to its good mechanical properties, functionally graded materials have become research hot spot[1,2]. It has been widely used in aerospace, mechanical engineering, biomedical engineering and other fields. Researchers on the dynamic buckling of functionally graded materials plate are explored Constantly[3,4]. Feldman and Aboudi[5] studied the linear dynamic buckling of functionally graded plates under in-plane loading. Shen [6] used the quadratic perturbation method to solve the nonlinear bending problem of simply-supported functionally graded material plates under the coupling condition of thermo and mechanical. The results show that the changes of volume fraction index and temperature have significant influence on the nonlinear bending behavior of the plate. Najafizadeh and Eslami[7,8] discussed the thermal buckling of circular plate in the in-plane load under simply supported and clamped supported conditions.

In this paper, Vogit's strain assumption, linear mixing rate and Kirchhoff sheet theory are used to derive the governing equations with Hamilton's principle. The expression of critical load for the functionally graded material plate is obtained. The expressions are numerically calculated by METLAB software programming. The influences of critical length, gradient index, material properties and boundary conditions for critical load are discussed.

\section{The governing equation of functionally graded material plates}

\footnotetext{
* Corresponding author email:wx2015tyut $@ 126 . c o m$
} 


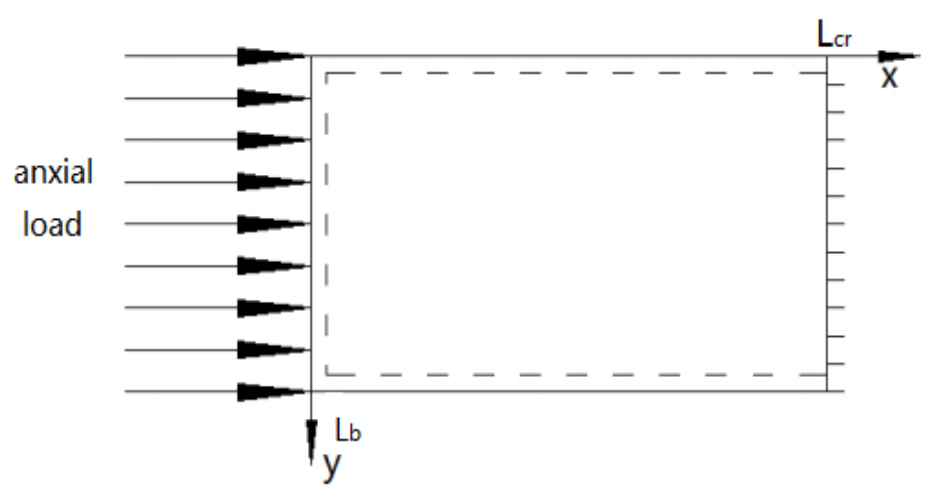

Figure 1 The plate with the length Lcr, width $\mathrm{Lb}$

The boundary conditions of one edge fixed and three edges simply supported can be written as

$$
\left\{\begin{array}{l}
w(0)=w_{x x}(0)=w\left(L_{c r}\right)=w_{x}\left(L_{c r}\right)=0 \\
w(0)=w_{y y}(0)=w\left(L_{b}\right)=w_{y y}\left(L_{b}\right)=0
\end{array}\right.
$$

The material properties of functionally graded material along the z-direction can be written as

$$
\left\{\begin{array}{l}
E_{(z)}=\left(E_{c}-E_{m}\right)\left(\frac{2 z+h}{2 h}\right)^{k}+E_{m} \\
\rho_{(z)}=\left(\rho_{c}-\rho_{m}\right)\left(\frac{2 z+h}{2 h}\right)^{k}+\rho_{m} \\
\mu_{(z)}=\left(\mu_{c}-\mu_{m}\right)\left(\frac{2 z+h}{2 h}\right)^{k}+\mu_{m}
\end{array}\right.
$$

$$
\begin{aligned}
T=\frac{1}{2} \int_{-\frac{h}{2}}^{\frac{h}{2}} \int_{0}^{L_{c r}} \int_{0}^{L_{b}} \rho_{(z)}\left[\left(\frac{\partial u}{\partial t}\right)^{2}+\left(\frac{\partial v}{\partial t}\right)^{2}+\left(\frac{\partial w}{\partial t}\right)^{2}\right] d x d y d z \\
=\frac{1}{2} \int_{0}^{L_{c r}} \int_{0}^{L_{b}}\left[I_{0}\left(\frac{\partial u_{0}}{\partial t}\right)^{2}+I_{0}\left(\frac{\partial v_{0}}{\partial t}\right)^{2}+I_{0}\left(\frac{\partial w_{0}}{\partial t}\right)^{2}-2 I_{1} \frac{\partial u_{0}}{\partial t} \frac{\partial^{2} w_{0}}{\partial x \partial t}-2 I_{1} \frac{\partial v_{0}}{\partial t} \frac{\partial^{2} w_{0}}{\partial y \partial t}\right. \\
\left.+I_{2}\left(\frac{\partial^{2} w_{0}}{\partial x \partial t}\right)^{2}+I_{2}\left(\frac{\partial^{2} w_{0}}{\partial y \partial t}\right)^{2}\right] d x d y
\end{aligned}
$$

The constitutive equation of functionally graded material plates is

$$
\left\{\begin{array}{l}
N_{x} \\
N_{y} \\
N_{x y} \\
M_{x} \\
M_{y} \\
M_{x y}
\end{array}\right\}=\left[\begin{array}{cccccc}
A_{11} & A_{12} & A_{16} & B_{11} & B_{12} & B_{16} \\
A_{12} & A_{22} & A_{26} & B_{12} & B_{22} & B_{26} \\
A_{16} & A_{26} & A_{66} & B_{16} & B_{26} & B_{66} \\
B_{11} & B_{12} & B_{16} & D_{11} & D_{12} & D_{16} \\
B_{12} & B_{22} & B_{26} & D_{12} & D_{22} & D_{26} \\
B_{16} & B_{26} & B_{66} & D_{16} & D_{26} & D_{66}
\end{array}\right]\left[\begin{array}{l}
\varepsilon_{x}^{0} \\
\varepsilon_{y}^{0} \\
\gamma_{x y}^{0} \\
k_{x} \\
k_{y} \\
k_{x y}
\end{array}\right]
$$

Kinetic energy with moment of inertia is

The deformation energy is

$$
U=\frac{1}{2} \int_{0}^{L_{c r}} \int_{0}^{L_{b}}\left(N_{x} \varepsilon_{x}^{0}+N_{y} \varepsilon_{y}^{0}+N_{x y} \gamma_{x y}^{0}+M_{x} k_{x}+M_{y} k_{y}+M_{x y} k_{x y}\right) d x d y
$$

The energy of external power is

$$
W=\frac{1}{2} \int_{0}^{L_{c r}} \int_{0}^{L_{b}} N_{(t)}\left(\frac{\partial w_{0}}{\partial x}\right)^{2} d x d y
$$

The coefficients are

$$
\begin{array}{lll}
A_{11}=A_{22}=\int_{-\frac{h}{2}}^{\frac{h}{2}} \frac{E_{(z)}}{1-\mu_{(z)}^{2}} d z & A_{12}=A_{21}=\int_{-\frac{h}{2}}^{\frac{h}{2}} \frac{\mu_{(z)} E_{(z)}}{1-\mu_{(z)}^{2}} d z & A_{66}=\int_{-\frac{h}{2}}^{\frac{h}{2}} \frac{E_{(z)}}{2\left(1+\mu_{(z)}\right)} d z \\
B_{11}=B_{22}=\int_{-\frac{h}{2}}^{\frac{h}{2}} \frac{z E_{(z)}}{1-\mu_{(z)}^{2}} d z & B_{12}=B_{21}=\int_{-\frac{h}{2}}^{\frac{h}{2}} \frac{z \mu_{(z)} E_{(z)}}{1-\mu_{(z)}^{2}} d z & B_{66}=\int_{-\frac{h}{2}}^{\frac{h}{2}} \frac{z E_{(z)}}{2\left(1+\mu_{(z)}\right)} d z \\
D_{11}=D_{22}=\int_{-\frac{h}{2}}^{\frac{h}{2}} \frac{z^{2} E_{(z)}}{1-\mu_{(z)}^{2}} d z & D_{12}=D_{21}=\int_{-\frac{h}{2}}^{\frac{h}{2}} \frac{z^{2} \mu_{(z)} E_{(z)}}{1-\mu_{(z)}^{2}} & D_{66}=\int_{-\frac{h}{2}}^{\frac{h}{2}} \frac{z^{2} E_{(z)}}{2\left(1+\mu_{(z)}\right)} d z
\end{array}
$$


Functionally graded material plates have the isotropic mechanical properties

$$
A_{16}=A_{26}=B_{16}=B_{26}=D_{16}=D_{26}=0
$$

Generalized inertia is defined in Eq. 4:

$$
\left(I_{0}, I_{1}, I_{2}\right)=\int_{-\frac{h}{2}}^{\frac{h}{2}} \rho_{(z)}\left(1, z, z^{2}\right) d z
$$

$$
D_{11} w_{x x x x}+\left(2 D_{22}+4 D_{66}\right) w_{x x y y}+D_{11} w_{y y y y}+N_{(t)} w_{x x}-I_{0} \ddot{w}+I_{2} \ddot{w}_{x x}+I_{2} \ddot{w_{y y}}=0
$$

\section{Dynamic buckling analysis of functionally graded material plates}

In $y$ direction, set the buckling mode of functionally graded material plates is $\sin \frac{m \pi y}{l_{b}}$ [9], set

$$
w=X(x) T(t) \sin \frac{m \pi y}{L_{b}}
$$

Substituting Eq. 9 into Eq. 8, the following equations can be obtained:

$$
D_{11} X_{\mathrm{xux} T} T-\left(2 D_{12}+4 D_{66}\right) X_{x x T} \frac{m^{2} \pi^{2}}{L_{b}^{2}}+D_{11} X T \frac{m^{4} \pi^{4}}{L_{b}^{4}}+N_{(t)} X_{x x} T-I_{0} X T_{t t}+I_{2} X_{x x} T_{t t}-I_{2} X T_{t t} \frac{m^{2} \pi^{2}}{L_{b}^{2}}=0
$$
into:

Separating the variables, Eq.10 can be transformed

$$
\left\{\begin{array}{l}
X_{x x x x}+\alpha X_{x x}+\beta X+\lambda \xi X-\lambda \eta X_{x x}=0 \\
T_{t t}+\lambda T=0
\end{array}\right.
$$

The coefficients of Eq.11 are

$$
\begin{aligned}
& \alpha=\frac{N_{(t)}}{D_{11}}-\frac{\left(2 D_{22}+4 D_{66}\right) m^{2} \pi^{2}}{D_{11} L_{b}{ }^{2}} \quad \beta=\frac{m^{4} \pi^{4}}{L_{b}{ }^{4}} \\
& \xi=\frac{I_{2}}{D_{11}} \quad \eta=\frac{I_{0}}{D_{11}}+\frac{I_{2} m^{2} \pi^{2}}{D_{11} L_{b}{ }^{2}}
\end{aligned}
$$

The solution of Eq. 11 is divergent when $\lambda<0$, the system is not stable and the dynamic buckling will occurs[10]. So the Eq. 11 has the dynamic buckling solution, the parameter $\lambda$ of Eq. 11 must satisfy the following conditions

$$
\lambda<0,(\alpha-\lambda \xi)^{2}-4(\beta-\lambda \zeta)>0
$$

The dynamic buckling solution to Eq. 13 is

$$
X(x)=C_{1} \sin k_{1} x+C_{2} \cos k_{1} x+C_{3} \sin k_{2} x+C_{4} \cos k_{2} x
$$

The coefficients of Eq.14 are

$$
\left\{\begin{array}{l}
k_{1}=\sqrt{\frac{\alpha-\lambda \xi+\sqrt{(\alpha-\lambda \xi)^{2}-4(\beta+\lambda \eta)}}{2}} \\
k_{2}=\sqrt{\frac{\alpha-\lambda \xi-\sqrt{(\alpha-\lambda \xi)^{2}-4(\beta+\lambda \eta)}}{2}}
\end{array}\right.
$$

Substituting Eq.3, Eq.4, Eq.5, Eq.6 and Eq.7 into Hamilton's principle $\quad \delta \int_{t_{2}}^{t_{1}}(T-U+W) d t=0 \quad$, the governing equation of functionally graded material plates can be written in the following form through calculation and simplification

Substituting Eq. 14 into Eq. 1, the linear equation group can be obtained

$$
\left[\begin{array}{cccc}
0 & 1 & 0 & 1 \\
0 & -k_{1}^{2} & 0 & -k_{2}^{2} \\
\sin k_{1} L_{c r} & \cos k_{1} L_{c r} & \sin k_{2} L_{c r} & \cos k_{2} L_{c r} \\
k_{1} \cos k_{1} L_{c r} & -k_{1} \sin k_{1} L_{c r} & k_{2} \cos k_{2} L_{c r} & -k_{2} \sin k_{2} L_{c r}
\end{array}\right]\left[\begin{array}{l}
C_{1} \\
C_{2} \\
C_{3} \\
C_{4}
\end{array}\right]=0
$$

The conditions of Eq. 15 with non-zero solution are $k_{1} l=n_{1} \pi \quad, \quad k_{2} l=n_{2} \pi \quad$ [10], where $n_{1}=1,2,3(1,2,3 \cdots), n_{2}=n_{1}+1$

The following forms can be obtained

$$
\left\{\begin{array}{l}
k_{1}^{2}+k_{2}^{2}=\frac{\pi^{2}\left(n_{1}^{2}+n_{2}^{2}\right)}{L_{c r}{ }^{2}}=\partial-\lambda \xi \\
k_{1}^{2} k_{2}^{2}=\frac{n_{1}^{2} n_{2}^{2} \pi^{4}}{L_{c r}{ }^{4}}=\beta+\lambda \eta
\end{array}\right.
$$

Simplifying Eq.16, the expression of critical dynamic buckling load $N_{c r}$ is

$$
N_{c r}=\frac{D_{11} \pi^{2}\left(n_{1}^{2}+n_{2}^{2}\right)}{L_{c r}{ }^{2}}+\frac{D_{11} \pi^{4} n_{1}^{2} n_{2}^{2} I_{2}}{L_{c r}{ }^{4}\left(I_{0} L_{b}^{2}+I_{2} m^{2} \pi^{2}\right)}-\frac{D_{22} m^{4} \pi^{4} I_{2}}{L_{b}^{2}\left(I_{0} L_{b}^{2}+I_{2} m^{2} \pi^{2}\right)}+\left(2 D_{12}+4 D_{66}\right) \frac{m^{2} \pi^{2}}{L_{b}{ }^{2}}
$$

Using the boundary conditions and the constraints conditions Eq. 1 and Eq.14 the buckling mode can be obtained

$$
w=T_{(r)} C_{1}\left[\left(\frac{n_{2} \sin \frac{n_{1} \pi}{L_{c r}}-n_{1} \sin \frac{n_{2} \pi}{L_{c r}}}{n_{2} \cos \frac{n_{2} \pi}{L_{c r}}-n_{1} \cos \frac{n_{1} \pi}{L_{c r}}}\right)\left(\cos \frac{n_{1} \pi x}{L_{c r}}-\cos \frac{n_{2} \pi x}{L_{c r}}\right)+\sin \frac{n_{1} \pi x}{L_{c r}}-\frac{n_{1}}{n_{2}} \sin \frac{n_{2} \pi x}{L_{c r}}\right] \sin \frac{m \pi y}{L_{b}}
$$

\section{Numerical results and discussion}

Table 1 Material parameters of functionally gradient materials plates

\begin{tabular}{cccc}
\hline $\begin{array}{c}\text { material } \\
\mathrm{s}\end{array}$ & $\begin{array}{c}\text { Elasticity Modulus } \\
E(\mathrm{GPa})\end{array}$ & $\begin{array}{c}\text { Density } \\
\rho\left(\mathrm{g} / \mathrm{cm}^{3}\right)\end{array}$ & $\begin{array}{c}\text { Poisson's Ratio } \\
\mu\end{array}$ \\
\hline $\begin{array}{c}\text { Ceramic } \\
\mathrm{s}\end{array}$ & 385 & 3.96 & 0.23 \\
$\begin{array}{c}\text { Titaniu } \\
\mathrm{m}\end{array}$ & 108.5 & 4.54 & 0.41 \\
Iron & 155 & 7.86 & 0.291 \\
Copper & 119 & 8.96 & 0.326 \\
\hline
\end{tabular}

The relationship between the critical load $L c r$ and critical length $\mathrm{Ncr}$ in different situations can be acquired 
and different orders of the buckling modes can be obtained by MATLAB.

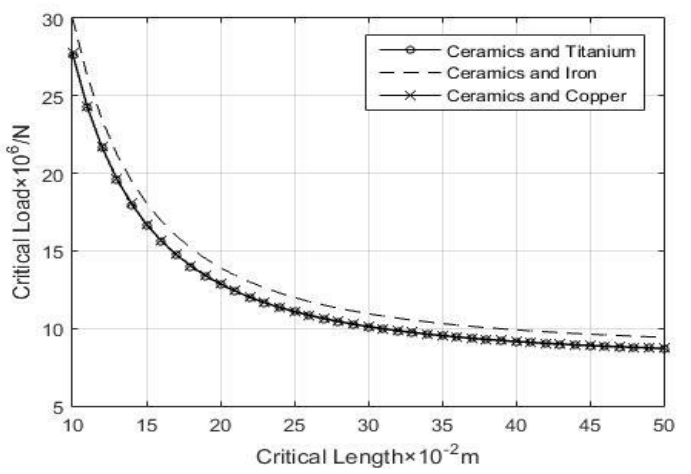

Figure 1. Relationship between the critical load and critical length when material is different

The critical load $\mathrm{Ncr}$ decreases exponentially with the increase of the critical length Lcr. Fig. 1 shows with Table 1 that the change of the elastic modulus of the material has obvious influence on the critical load of dynamic buckling of the functionally graded material plate, and the influence of Poisson's ratio is relatively small.

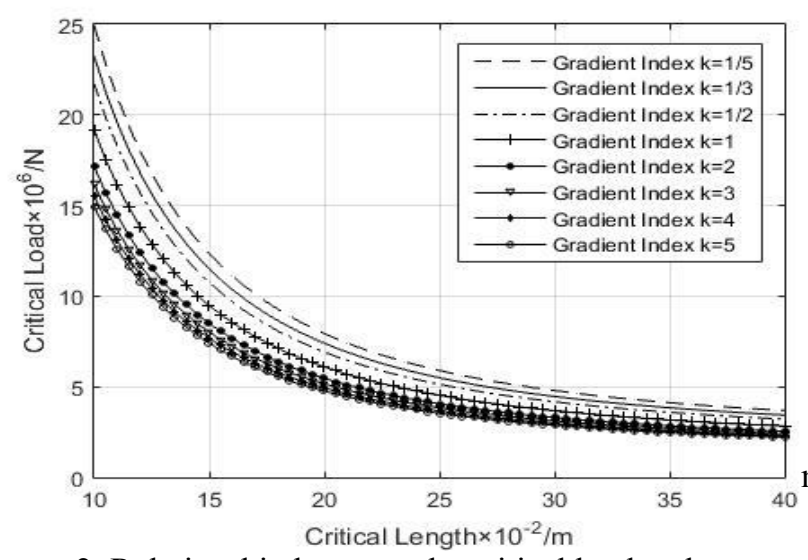

Figure 2. Relationship between the critical load and length when gradient index is different

The critical load $\mathrm{Ncr}$ decreases with the increase of gradient index $k$. The relationship between $\mathrm{Ncr}$ and $\mathrm{Lcr}$ changed obviously when the value of $k$ is small and the change of $k$ value in the range of (0-1) has great impact on $N c r$. The critical load curve corresponding to different $k$ values tends to level with the increase of $L c r$.

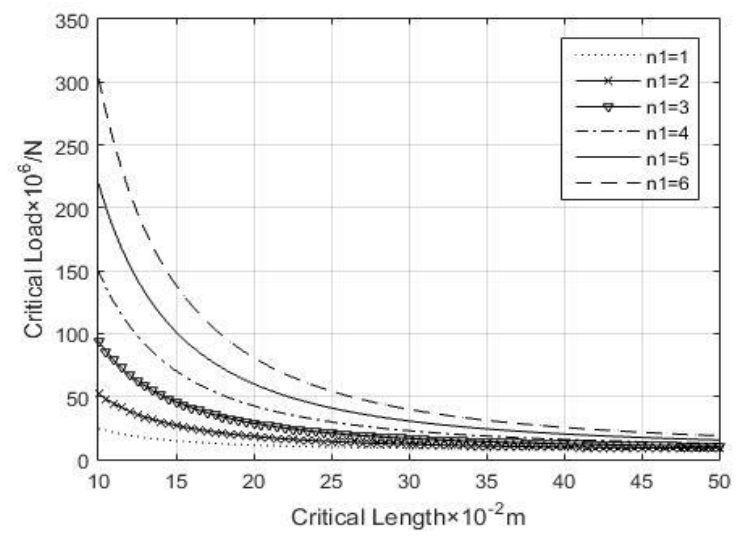

Figure 3. Relationship between critical load and length when the modal number of $\mathrm{X}$ direction is different
The critical load $N c r$ increases with the increase of modal number $n 1$. The change of $\mathrm{Ncr}$ with $\mathrm{Lcr}$ is more obvious in the condition of high value of $n l$
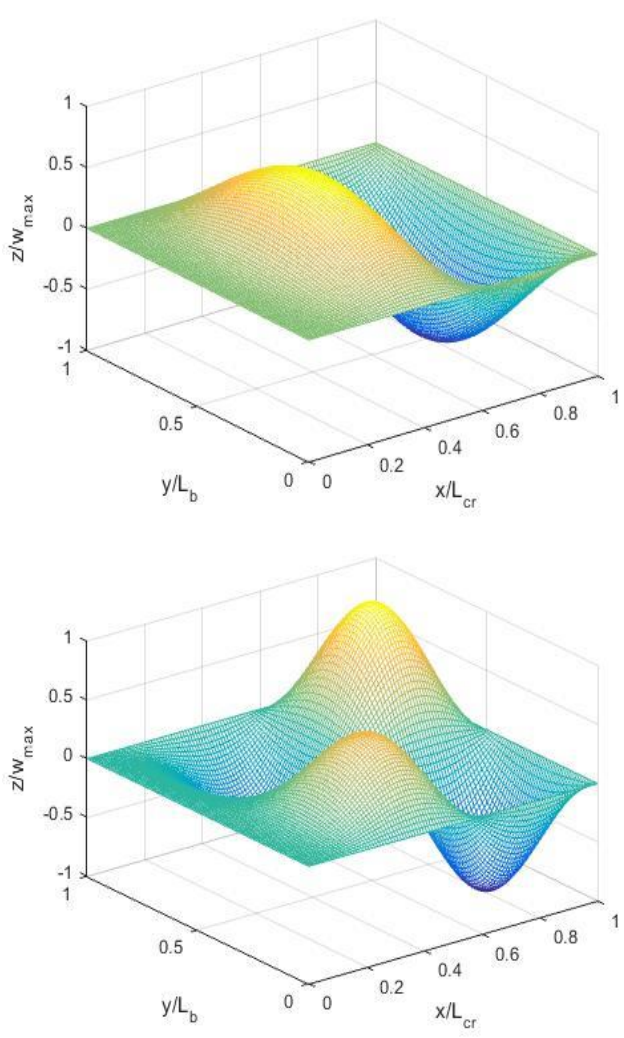

$$
\begin{aligned}
& \text { (a) } n_{1}=2, m=1 \\
& \text { (b) } n_{1}=2, m=2
\end{aligned}
$$

Figure 4. Different orders of the buckling modes

The amplitude modes $w$ of functionally graded material plates increasing positively in $\mathrm{X}$ direction.

\section{Conclusion}

1. The analytical expression of dynamic buckling critical load (17) and buckling modal expression (18) of functionally graded material plates are obtained. The buckling modes are shown in Fig.3.

2. Calculate Eqs. (17) and (18) by MATLAB, and discuss the influence of critical length, gradient index, material property and order of buckling modes on the critical buckling load and buckling mode. The results show that the critical load of dynamic buckling decreases exponentially with the increase of length, and the critical load of buckling decreases with the increase of gradient index $k$. The gradient index $k$ have great influence on buckling critical load in conditions that the gradient index $k$ changes in the range of $(0-1)$. The buckling critical load is greatly affected by the elastic modulus and the boundary conditions have great influence on the buckling mode of the functionally graded material plates.

\section{Acknowledgements}

The project is supported by Science Foundation for 
Young Scholars of Shanxi Province of China (NO.201601D021127) and the Graduate Educational Reformation of Shanxi Province of China (NO.2015JG41)

\section{References}

1. $\mathrm{Na} \mathrm{K} \quad \mathrm{S}, \mathrm{Kim} \quad \mathrm{J} \quad \mathrm{H}$. Three-dimensional thermomechanical buckling analysis for functionally graded composite plates[J]. Composite Structures, 2006, 73(4):413-422.

2. Huang X L, Shen H S. Nonlinear vibration and dynamic response of functionally graded plates in thermal environments[J]. International Journal of Solids \& Structures, 2005, 41(9-10):2403-2427.

3. Huang X L, Shen H S. Nonlinear vibration and dynamic response of functionally graded plates in thermal environments[J]. International Journal of Solids \& Structures, 2005, 41(9-10):2403-2427.

4. Shen H. Bending, buckling and vibration of functionally graded plates and shells $[\mathrm{J}]$. Advances in Mechanics, 2004, 34(01):53-60.

5. Feldman E, Aboudi J. Buckling analysis of functionally graded plates subjected to uniaxial
loading[J]. Composite Structures, 1997, 38(1-4):29-36.

6. Shen H S. Nonlinear bending response of functionally graded plates subjected to transverse loads and in thermal environments[J]. International Journal of Mechanical Sciences, 2002, 44(3):561-584.

7. Najafizadeh M M, Eslami M R. Buckling analysis of circular plates of functionally graded materials under uniform radial compression[J]. International Journal of Mechanical Sciences, 2002, 44(12):2479-2493.

8. Najafizadeh M M, Eslami M R. First-Order-Theory-Based Thermoelastic Stability of Functionally Graded Material Circular Plates[J]. Aiaa Journal, 2002, 40(7):1444-1450.

9. Leissa A W, Kang $\mathbf{J}$ H. Exact solutions for vibration and buckling of an SS-C-SS-C rectangular plate loaded by linearly varying in-plane stresses[J]. International Journal of Mechanical Sciences, 2002, 44(9):1925-1945

10. HAN Zhi-jun, Experimental and theoretic studies on the impact buckling of columns and the effect of stress wave [D].Taiyuan University of Technology, (2005). 